\title{
Impact of anaesthesia on lung function in children
}

\author{
ERS Annual Award for Paediatric Respiratory Research in Europe, sponsored by AstraZeneca
}

\author{
Britta S. von Ungern-Sternberg
}

Dept of Anaesthesia, Princess Margaret Hospital for Children and Division of Clinical Sciences, Telethon Institute for Child Health Research, Perth, Australia

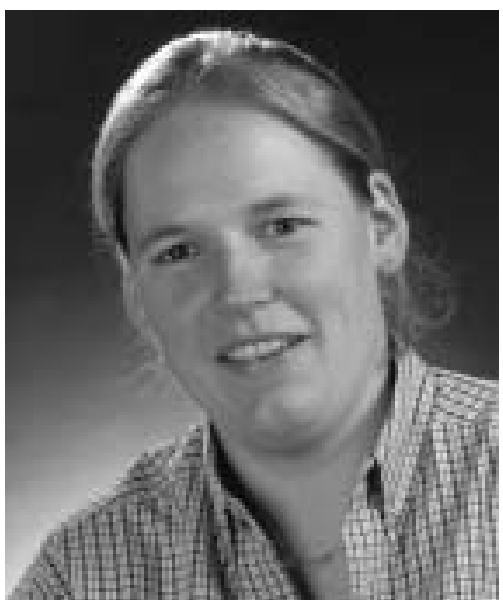

$\mathbf{R}$

espiratory adverse events are major causes of morbidity and mortality during paediatric anaesthesia. Several factors, such as asthma, bronchial hyperreactivity, upper respiratory tract infection or passive smoking, are known to increase the risk of respiratory complications. Since all these risk factors have a high prevalence in children, it is crucial for the paediatric anaesthesia practice to anticipate, recognise and treat common respiratory complications. The ability to prevent respiratory complications requires an understanding of the underlying physiology and pathophysiology, but until recently little has been known of the impact of anaesthesia and its different components (e.g. anaesthetic agents, ventilation strategies) on respiratory function in children. My research over the past years has focused on this area and, in particular, on the impact of different anaesthetic agents and regional anaesthesia techniques, as well as body positioning on functional residual capacity and ventilation homogeneity.

\section{IMPACT OF VENTILATION STRATEGIES ON RESPIRATORY FUNCTION}

Impact of positive end-expiratory pressure in anesthetised children exposed to high levels of inspired oxygen

The high fractions of inspired oxygen $\left(\mathrm{FI}, \mathrm{O}_{2}\right)$ that are commonly administered, especially at induction and termination of

STATEMENT OF INTEREST: None declared. anaesthesia, result in airway closure and, as a consequence, decreased functional residual capacity (FRC). Our study examined the effects of $\mathrm{FI}_{1} \mathrm{O}_{2} 0.3$ (in air) versus $\mathrm{FI}, \mathrm{O}_{2} 1.0$ and its reversibility by the application of continuous positive endexpiratory pressure (PEEP) of $6 \mathrm{cmH}_{2} \mathrm{O}(0.6 \mathrm{kPa})$ compared with PEEP of $3 \mathrm{cmH}_{2} \mathrm{O}(0.3 \mathrm{kPa})$ on FRC and ventilation distribution in preschool children under general anaesthesia [1]. An increase of $\mathrm{FI}_{1} \mathrm{O}_{2}$ from 0.3 to 1.0 with a PEEP of $3 \mathrm{cmH}_{2} \mathrm{O}(0.3 \mathrm{kPa})$ decreased the measured FRC and ventilation homogeneity, while FRC and ventilation homogeneity remained unchanged when $6 \mathrm{cmH}_{2} \mathrm{O}(0.6 \mathrm{kPa})$ PEEP was applied.

Impact of different breathing systems for manual ventilation Skilled use of bag and mask ventilation is of fundamental importance in paediatric anaesthesia. Although often considered as simple, there are a number of potential hazards resulting from inadequate manipulation that can jeopardise gas exchange. Consecutive gastric inflation can lead to increases in abdominal pressure, dilation of the oesophageal sphincter and subsequent regurgitation and broncho-aspiration. Furthermore, increasing amounts of air in the stomach can preclude effective ventilation and compromise respiratory function by inducing a cranial shift of the diaphragm and decreasing functional residual capacity.

For many years, the modified Jackson Rees T-piece system has been advocated as the best breathing system to ensure adequate ventilation in preschool children. Concerns regarding environmental pollution by anaesthetic agents as well as the development of low resistance circle systems contributed to a change in clinical practice from the Jackson Rees system to the circle system. Moreover, compared with the circle system, the Jackson Rees system requires a greater peak transpulmonary pressure to achieve the same peak expiratory flows, suggesting an increased resistance and, thus, increasing the risk for gastric insufflation.

The results of our study show that efficacy of bag and mask ventilation is highly dependent on the training of the anaesthetist in charge of ventilation [2]. The breathing system plays a crucial role in preventing ventilatory impairment as a result of gastric insufflation. As the Jackson Rees system requires a more skilled operator compared with the circle, the latter should be considered as first choice for teaching and in everyday clinical practice. 


\section{IMPACT OF DIFFERENT ANAESTHETIC AGENTS ON RESPIRATORY FUNCTION}

Impact of neuromuscular blockade on FRC and ventilation homogeneity in anaesthetised young infants and preschool children

Using an interdisciplinary approach, including paediatric anaesthetists, pulmonologists and paediatric intensivists, we assessed the impact of neuromuscular blockade and PEEP on FRC and ventilation homogeneity in young infants and preschool-aged children [3]. Neuromuscular blockade was found to have a deleterious effect on lung volume and ventilation distribution, particularly in young infants, because of their greater tendency to experience airway collapse; this decrease could be eliminated by the addition of a low PEEP of $6 \mathrm{cmH}_{2} \mathrm{O}(0.6 \mathrm{kPa})$. Sedation and anaesthesia were known to decrease FRC in patients of all ages, but this is the first study demonstrating the disproportionately high impact of neuromuscular blocking agents on respiratory function in young infants, a population particularly at the risk of hypoxaemia. The results of this study have changed clinical practice in our institution, as well as others, such that low levels of PEEP during paediatric anaesthesia are routinely used and neuromuscular blocking agents are avoided.

\section{Impact of depth of propofol anaesthesia}

Anaesthesia or deep sedation is frequently required in children because of their lack of cooperation with diagnostic procedures or minor interventions. Based on the invasiveness of the intervention (e.g. magnetic resonance imaging scanning, bronchoscopy), varying levels of anaesthesia are required to perform such procedures successfully. For deep sedation in children, propofol is a commonly used sedative agent. However, increasing doses of propofol can jeopardise upper airway patency by increasing airway collapsibility. In addition, our study showed that a deeper level of propofol sedation reduced FRC and ventilation distribution in the preschoolaged children, suggesting an increasing vulnerability to hypoxaemia with increasing doses of propofol [4].

\section{Impact of depth of ketamine anaesthesia}

Ketamine is a commonly used sedation agent, particularly in the emergency setting or when anaesthesia is performed by nonanaesthetists because of its unique analgesic and hypnotic properties that maintain spontaneous breathing. In contrast to most anaesthetic agents, ketamine has no muscle relaxant properties. This is also reflected in the finding of our study [5] that a deeper level of ketamine anaesthesia does not result in significant changes in FRC, ventilation distribution and minute ventilation in otherwise healthy, preschool-aged children indicating that pulmonary function is not impaired by a deeper level of ketamine anaesthesia.

\section{Impact of sevoflurane and desflurane in children with and without susceptible airways}

Among the risk factors that increase perioperative respiratory complications, bronchial hyperreactivity is the most frequent underlying pathophysiological condition encountered in paediatric anaesthesia. During the last decade, the use of halothane has been gradually replaced by sevoflurane and desflurane because of their coefficients of blood solubility and decreased side-effects. Our study provides objective and systematic measurements of airway and respiratory tissue mechanics in children with normal lungs and those with a potential for airway hyperresponsiveness under these two agents [6]. Our results indicate that the bronchodilatory ability of sevoflurane is independent of the clinical status of the child, whereas the bronchoconstricting effects of desflurane were apparent in all children, although with a markedly exaggerated airway narrowing in the presence of susceptible airways. Thus, the use of desflurane should be avoided in the anaesthesia of children who exhibit a clinical history of recent upper airway infection or any other pulmonary disease that may be associated with bronchial hyperreactivity.

\section{Impact of caudal block}

Muscle relaxation induced by an epidural anaesthesia with bupivacaine via the caudal route decreases abdominal muscle tone while sparing the muscles of the respiratory system (diaphragm, intercostal muscles) and, therefore, interacts with the balance between chest wall and lung recoil pressure and tension of the diaphragm, which thus determines the static resting volume of the lung. The results of a randomised, controlled trial of ours showed a significant increase in FRC and an improvement in ventilation homogeneity after caudal block [7]. Under propofol sedation, FRC and ventilation distribution remained unchanged in the control group during the 30-min study period. Our results suggest that caudal block might have a beneficial impact on respiratory physiology in preschool children under general anaesthesia.

\section{IMPACT OF BODY POSITIONING ON RESPIRATORY FUNCTION \\ Impact of different prone positioning techniques}

The prone position has been advocated as a therapeutic measure in order to improve oxygenation. Although since its first description by BRYAN [8], numerous studies have reported conflicting findings regarding the effect of the prone position on pulmonary function. While prone positioning improves arterial oxygen saturation, the results regarding its effect on FRC have been controversial. Therefore, additional confounding factors, such as changes in ventilation distribution or differences in positioning techniques, might be important. In our recent study, we demonstrated that the augmented prone position (gel pads supporting the shoulders and the pelvis), in contrast to the flat prone position, increases FRC and thus could help to optimise respiratory function in paralysed, mechanically ventilated, healthy children [9].

\section{Impact of neuromuscular blockade and lateral position}

General anaesthesia, particularly with neuromuscular blockade, decreases FRC and ventilation homogeneity [3]. These reductions can decrease the end-expiratory lung volume below the closing capacity resulting in airway closure, absorption collapse of the lungs and shunting. Various surgical interventions require body positioning different from the supine position. There is an increase in FRC in children in the lateral position compared with the supine position, perhaps caused by a decrease of intra-abdominal pressure on the diaphragm in the lateral position. It is expected that the effect of neuromuscular blockade on FRC and ventilation homogeneity in the lateral position might differ from that in the supine position. Our study [10] showed that neuromuscular blockade significantly reduced 
FRC and decreased ventilation homogeneity in anaesthetised children in the lateral position. The addition of PEEP $\left(3 \mathrm{cmH}_{2} \mathrm{O}\right.$ $(0.3 \mathrm{kPa}))$ effectively restored the effects of neuromuscular blockade on FRC and ventilation homogeneity; its use could also prove to be beneficial for anaesthetised, paralysed children in the lateral body position.

\section{Impact of Trendelenburg positioning}

Trendelenburg positioning, as defined by a head-down tilt, is routinely used in anaesthesia when inserting a central venous catheter to enhance the calibre of the jugular or subclavian veins and to prevent an air embolism. This procedure is also performed in critically ill children in whom gas exchange might already be impaired. Moreover, in adults, the headdown tilt position can reduce lung function and impair gas exchange. Children, in general, and children with cardiopulmonary disease, in particular, might be prone to hypoxaemia after a further decrease in respiratory function after headdown positioning, as central venous catheter positioning, per se, is associated with a high incidence of morbidity and mortality in paediatric anaesthesia. In our study, head-down tilt position was found to lead to a significant decrease in FRC and an increase in ventilation inhomogeneity [11]. These changes in lung function are not restored after simple supine repositioning suggesting airway closures that necessitate a recruitment manoeuvre. Therefore, anaesthetists must be aware of the impact of head-down tilt positioning on lung volume and gas exchange and should consider using a recruitment manoeuvre to restore baseline lung volumes.

\section{IMPACT OF SURGERY ON RESPIRATORY FUNCTION Impact of cardiopulmonary bypass and aortic clamping in children undergoing repair of congenital heart disease}

Many factors impair lung function after cardiopulmonary bypass. For example, cardiopulmonary bypass affects lung function by inducing an inflammatory response or promoting interstitial water extravasation. In addition, congenital heart disease is often associated with an impaired pulmonary haemodynamic condition that has a major impact on the mechanical properties of the lung. In spite of improved perioperative ventilation strategies in children with congenital heart disease, their ventilatory pattern and performance remains impaired in the post-operative period, enhancing the ventilation perfusion mismatch. All these phenomena lead to airway closure, atelectasis, decrease in FRC and consecutive hypoxaemia.

The tethering effect exerted by pulmonary capillary perfusion pressure on the alveolar wall is a pathophysiological mechanism responsible for airway closure that contributes to alveolar stability and greatly affects the mechanical properties of the lung. Our most recent study demonstrates the important role of both chest and alveolar wall configurations for maintaining an open lung and for determining lung resting volume and ventilation distribution [12]. While opening of the chest wall improves alveolar recruitment via changes in transpulmonary pressure, blood flow appears essential for alveolar stability.

\section{THE IMPACT OF MY WORK ON CLINICAL OR RESEARCH PRACTICE}

In children, one-third of the anaesthesia-related mortality and three-quarters of all critical incidences are caused by respiratory problems. Thus, understanding the changes in lung function caused by the different components of anaesthetic management leads to the improvement of paediatric anaesthesia and increases safety for children undergoing anaesthesia or sedation. This series of studies further characterises the impact of different anaesthetic agents, positioning techniques, ventilation and surgery on lung function, and thus helps to optimise the conduct of anaesthesia with regard to lung function changes in the paediatric population.

\section{MY RESEARCH AS PART OF MY WORKING GROUP/ RESEARCH TEAM}

I work within an interdisciplinary, international research team consisting of paediatric anaesthetists, paediatric respiratory physicians, paediatric intensivists and specialists in biomedical informatics and engineering. Our group consists of the following members: at the University Children's Hospital, Basel, Switzerland, are Dr T.O. Erb and Professor F.J. Frei (Division of Paediatric Anaesthesia), and Professor J. Hammer (Dept of Pulmonology and Intensive Care Medicine); at the University Hospitals, Geneva, Switzerland, is Dr W. Habre (Paediatric Anaesthesia Unit); at the University of Szeged, Hungary, are Professor Z. Hantos and Associate Professor F. Petak (Dept of Medical Informatics); and at the Mater Misericordiae Hospital Brisbane, Australia, is Associate Professor A. Schibler (Dept of Paediatric Intensive Care).

The work described above was conducted at the Division of Paediatric Anaesthesia, University Children's Hospital of Basel, Switzerland, and at the Paediatric Anaesthesia Unit, University Hospitals Geneva, Switzerland.

Our main research focus is the impact of anaesthesia on lung function and the development of strategies to prevent respiratory complications in the perioperative period. The interdisciplinary approach allows for integrating different specialist knowledge into our study design and broadening of our research horizon.

At present, a fellowship from the Swiss Foundation for Grants in Biology and Medicine, in cooperation with the Swiss National Science Foundation, allows me to broaden my knowledge of respiratory medicine and paediatric anaesthesia at the Telethon Institute for Child Health Research and the Princess Margaret Hospital for Children in Perth, Australia. In addition, the research performed in Australia also reflects my ongoing collaborations with the team members in Basel and Geneva, Switzerland and Szeged, Hungary.

\section{REFERENCES}

1 von Ungern-Sternberg BS, Regli A, Schibler A, Hammer J, Frei FJ, Erb TO. Impact of positive end-expiratory pressure on functional residual capacity and ventilation homogeneity impairment in anesthetized children exposed to high levels of inspired oxygen. Anesth Analg 2006; 104: 1364-1368.

2 von Ungern-Sternberg BS, Saudan S, Regli A, Schaub E, Erb TO, Habre W. Should the modified Jackson Rees Ayres-T piece breathing system be abandoned in preschool children? Paediatr Anaesth 2007; 17: 654-660.

3 von Ungern-Sternberg BS, Hammer J, Schibler A, Frei FJ, Erb TO. Decrease of functional residual capacity and 
ventilation homogeneity following neuromuscular blockade in anesthetized young infants and preschool children. Anesthesiology 2006; 105: 670-675.

4 von Ungern-Sternberg BS, Frei FJ, Hammer J, Schibler A, Doerig R, Erb TO. Impact of depth of propofol anaesthesia on the functional residual capacity and ventilation distribution in healthy preschool children. Br J Anaesth 2007; 98: 503-508.

5 von Ungern-Sternberg BS, Regli A, Frei FJ, et al. A deeper level of ketamine anesthesia does not affect functional residual capacity and ventilation distribution in healthy preschool children. Paediatr Anaesth 2007; 17: 1150-1155.

6 von Ungern-Sternberg BS, Saudan S, Petak F, Hantos Z, Habre W. Desflurane but not sevoflurane impairs airway and respiratory tissue mechanics in children with susceptible airways. Anesthesiology 2008; 108: 216-224.

7 von Ungern-Sternberg BS, Regli A, Frei FJ, Hammer J, Schibler A, Erb TO. The effect of caudal block on functional residual capacity and ventilation homogeneity in healthy children. Anaesthesia 2006; 61: 758-763.

8 Bryan AC. Conference on the scientific basis of respiratory therapy. Pulmonary physiotherapy in the paediatric age group. Comments of a devil's advocate. Am Rev Respir Dis 1974; 110: 143-144.

9 von Ungern-Sternberg BS, Hammer J, Frei FJ, Jordi Ritz EM, Schibler A, Erb TO. Prone equals prone? Impact of positioning techniques on respiratory function in anesthetized and paralyzed healthy children. Intensive Care Med 2007; 33: 1771-1777.

10 von Ungern-Sternberg BS, Regli A, Frei FJ, et al. Decrease of functional residual capacity and ventilation homogeneity after neuromuscular blockade in anesthetized preschool children in the lateral position. Paediatr Anaesth 2007; 17: 841-845.

11 Regli A, Habre W, Saudan S, Mamie C, Erb TO, von UngernSternberg BS. Impact of Trendelenburg positioning on functional residual capacity and ventilation homogeneity in anaesthetised children. Anaesthesia 2007; 62: 451-455.

12 von Ungern-Sternberg BS, Petak F, Saudan S, Pellegrini M, Erb TO, Habre W. Impact of cardiopulmonary bypass and aortic clamping on functional residual capacity and ventilation distribution in children. J Thoracic Cardiovasc Surg 2007; 134: 1193-1198. 